\title{
Cranial Volume and Occipito-Frontal Circumference in Neonates
}

\author{
KORAVANGATTU SANKARAN ${ }^{(10)}$ LLOYD WALTON, ZANE TYMCHAK, SUSAN HAYTON, \\ ELIZABETH DUFF, LEONARD TAN, AND THOMAS B. BEST \\ Perinatal Research Laboratory, Newborn Services, Department of Pediatrics and Social and Preventative \\ Medicine, College of Medicine, University of Saskatchewan, Saskatoon, Canada
}

\begin{abstract}
Summary
Occipitofrontal head circumference (OFC), using tape, and calibrated scale and cranial volumes, using styrofoam plastic cast, were measured in nine preterm and 13 term infants. Term infants had a mean \pm S.E. gestation of $40 \pm 0.2$ wk and a mean \pm S.E. birth weight of $3461 \pm 108 \mathrm{~g}$. Preterm infants had a mean \pm S.E. gestation of $30.5 \pm 1.4 \mathrm{wk}$ and a mean \pm S.E. birth weight of $1596 \pm 211 \mathrm{~g}$. Five sequential measurements were made on term infants and nine such measurements were done on preterm infants at specified time intervals. The values of cranial volume were compared with previously published results in term infants. Brain weight and cranial volumes were also compared in $\mathbf{1 0}$ preterm infants and seven term infants who died of non-intracranial pathology. To determine if this method is reproducible, it was tested using 50 paired measurements and was found to be within $3 \%$. There was a significant decrease in cranial volume in term and preterm infants with time. Term infants reached the control value at $64 \mathrm{~h}$ of age and preterm infants reached the control value at $160 \mathrm{~h}$ of age. Preterm infants showed a significant decrease in OFC but term infants did not. The rate of change was not significantly different in either group. We conclude, (I) cranial volume can be measured accurately and are reproducible in term and preterm infants by non-invasive means; (2) OFC decreases about $2 \%$ in preterm infants by 1 wk and cranial volume decreases by $5 \%$ in term infants and $7 \%$ in preterm infants in the first week of life; and (3) OFC is not a reliable method of measurement of brain growth in term neonates in the first week of life.
\end{abstract}

\section{Abbreviations}

Cranial volume, neurocranial-scalp volume OFC, occipitofrontal head circumference

At birth, newborn infants are suddenly exposed to an extrauterine environment. In order to adjust to the new environs, dramatic shifts take place among the various fluid compartments in the body (6). Decrease in head size with the calvarial bones overriding the suture is a clinical example demonstrating such a phenomenon during the first few days of life (8). This so called "postnatal head shrinkage" in infants has been assessed previously by various means such as calculation of cranial volume by measurements of OFC, tri-radial diameter measurement of the head using ultrasound, by caliper measurement, and by radiographic techniques $(1,2,5,7)$. In all of these studies cranial volumes have been calculated, not measured directly.

Measurements of cranial volume during the first few days of life are still not available. The purpose of this study was to sequentially measure cranial volume in term and preterm infants as well as OFC measurements.

\section{SUBJECTS AND METHODS}

OFC and cranial volumes were measured in two groups of infants. Group 1 was composed of nine "healthy" preterm infants with a mean gestation of $30.5 \pm 1.4 \mathrm{wk}$ (range, 26-35) and a mean birth weight of $1596 \pm 211 \mathrm{~g}$ (range, 890-2460). Group 2 consisted of 13 healthy term infants with a mean gestation of 40 $\pm 0.2 \mathrm{wk}$ (range, 39-42) and a mean birth weight of $3461 \pm 108$ $\mathrm{g}$ (range, 3180-4250). All values are expressed with mean \pm S.E. Three infants in Group 1 were born by cesarean section. No infant had any overt scalp edema and none were subjected to scalp vein infusion. Intracranial pathology was excluded using complete clinical and neurologic examination along with normal brain imaging. All preterm infants were breathing spontaneously without any clinical problems and being fed orally with supplemental intravenous infusions whenever necessary. Infants were cared for using standard recommenced procedures (4). All had head circumferences between the 25-75th percentile for their age and sex. The initial OFC and cranial volume were measured at $24 \mathrm{~h}$ of age and twice daily thereafter. The first measurement was delayed until $24 \mathrm{~h}$ of age to minimize the compounding problem of scalp edema. The OFC was measured with a nonstretchable fiberglass tape while holding the head in the lateral position. Marks were made on the tape and later measured against standard calibrated steel scale (RTC C500, Tokyo, Japan). Duplicate measurements were made by two investigators.

Cranial volume measurements. Cranial volume measurements were carried out using a non-invasive technique as described below.

A cellophane bag lined with $0.5-\mathrm{mm}$ diameter styrofoam pellets was made securely airtight and connected to a wall suction. Using minimal suction (10-20 $\mathrm{mmHg}$ ), pellets were spread inside the bag and later wrapped around the baby's head as shown in Figure 1. Landmarks used were the upper margins of both ears (where pinnas join the scalp superiorly), the eyebrows, and the inferior margin of the occiput. The suction was then increased to high $(200 \mathrm{mmHg})$. Care was taken to have the bag securely on the scalp and then the plastic cast was removed from the head (Fig. 2). Next a thin polythene sheet was placed inside the cast to serve as a waterproof liner and the cast was completely filled with water. The volume of water was then measured carefully. Fifty such measurements were made on normal infants in order to establish the reliability of this method. We found that using this technique, different individuals could, after practice, obtain values for an infant's cranial volumes that were consistently within $3 \%$ of one another $(P<0.001)$. The calculated cranial volumes in normo-cephalic infants were compared with our values. In an attempt to validate the technique further, the brain weight at autopsy of seven term infants and eight preterm infants was compared with measured skull volume. It was assumed that the specific gravity of brain was 1.0. Again the values were within $3 \%$ of one another. 

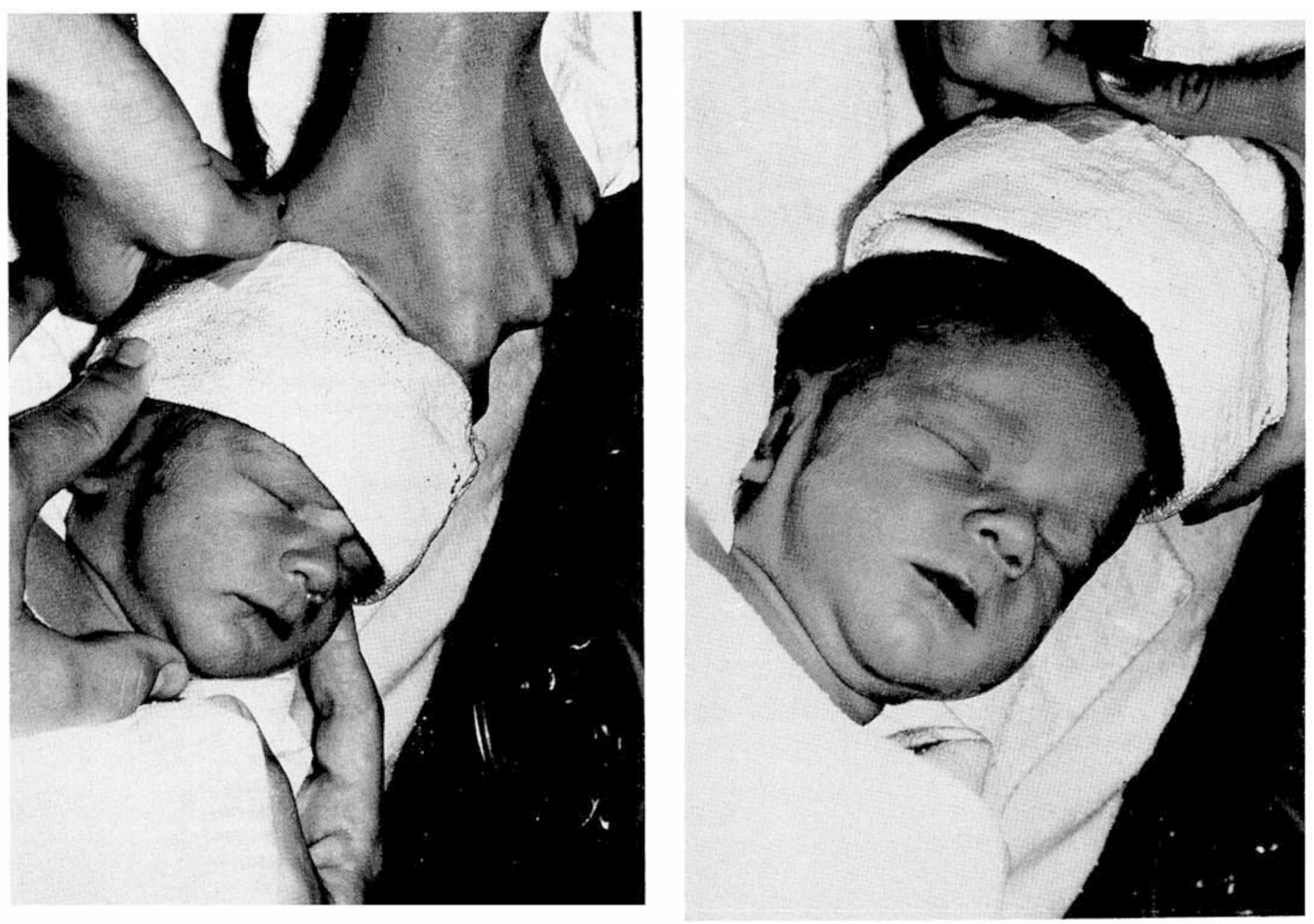

Fig. 1. Technique of application of styrofoam plastic case using landmarks as described in text.

\section{RESULTS}

Figure 3 shows the cranial volume and OFC measurements in term and preterm infants with mean and standard error. Figures 4 and 5 show the relative changes in cranial volume and head circumference in term and preterm infants. The data was analyzed by ANOVA blocking on the subjects. One could look upon this technique as a logical extension of the $t$ test. The two-way ANOVA consisted of 13 rows and 5 columns in Group II. A similar technique of analysis was used in Group I. There was a significant decrease in cranial volume in Group 1 over time $(P$ $=0.0049$ ). The corresponding head circumference also showed a significant decrease $(P=0.0026)$. In Group II there was a significant decrease in cranial volume $(P=0.0001)$; however, there was no difference in OFC. The rate of decrease in cranial volume in both groups was also tested but not found to be significant. Note that in Group II the cranial volume had reached the control values at about $64 \mathrm{~h}$ of age. Group I took longer (160 h) to reach control values. Term infants (Group II) decreased about $5 \%$ of volume whereas preterm infants lost $7 \%$ of their volume. There was no significant differences between brain weight and cranial volume in either group (term, $465 \pm 15$ versus $460 \pm 10$ and preterm, $240 \pm 20$ versus $246 \pm 26)(P<0.001)$.

\section{DISCUSSION}

To our knowledge this is the first report with quantitative measurements of cranial volume and occipitofrontal circumference using a non-invasive method. The reliability of our measurements was tested against calculated volumes for term infants,

Fig. 2. The plastic cast is being removed from the infant without causing any disturbance.

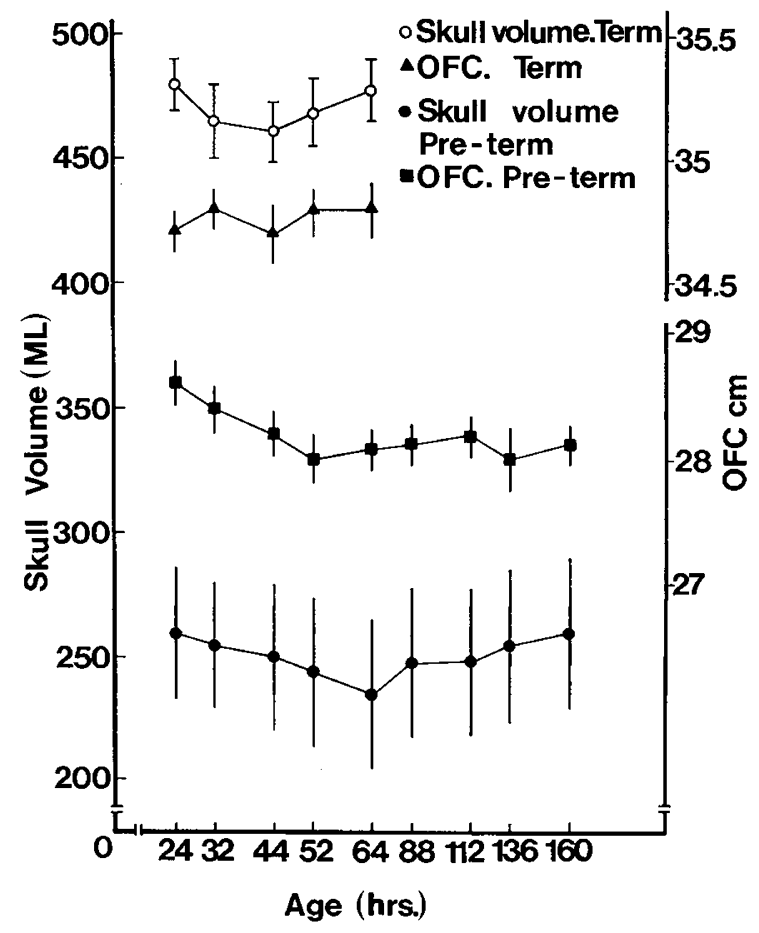

Fig. 3. Values of skull volume and OFC in term and preterm infants \pm S.E. 


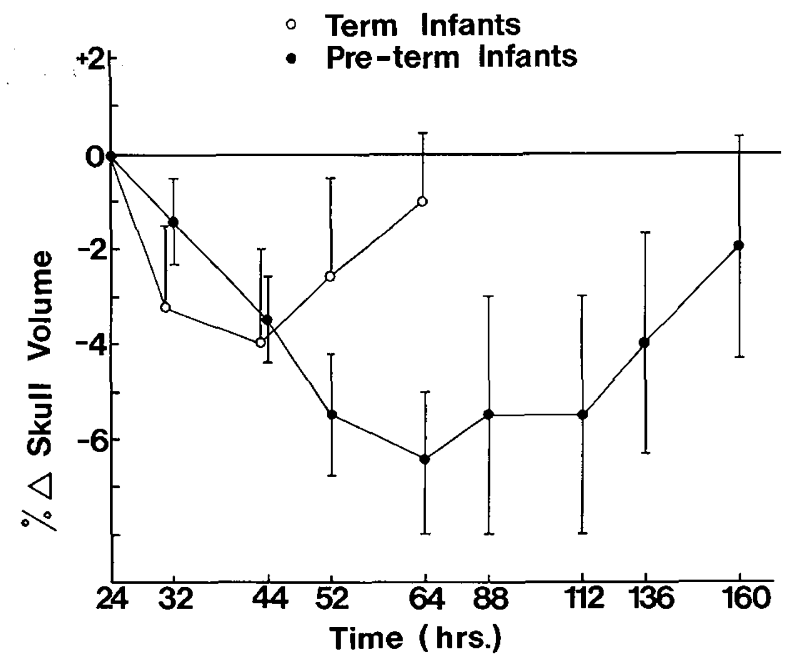

Fig. 4. \% change of skull volume \pm S.E. in term and preterm infants.

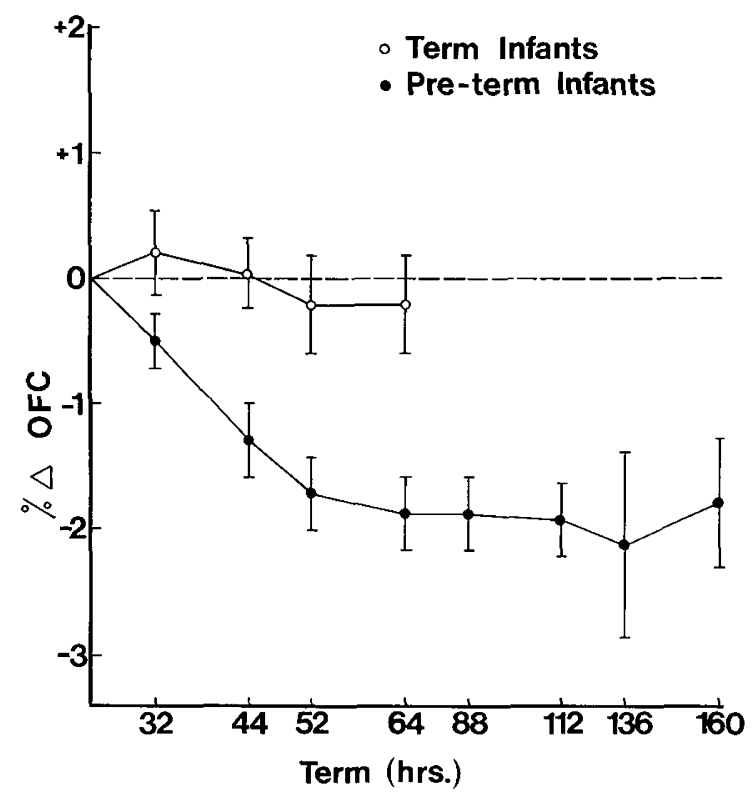

Fig. 5. \% change of $\mathrm{OFC} \pm$ S.E. in term and preterm infants.

and there was less than $3 \%$ difference in these measurements. We could not compare values of cranial volume measurements in preterm infants against calculated volumes because such data was not available in the literature. The method was also tested by measuring brain weight directly in autopsied infants. Again, the method gave results within $3 \%$ of that of the brain weight. We believe the consistency and accuracy of this measurement is dependable.

As the skull is the bony framework for both face and braincase (neurocranium) we tried to measure the internal volume of neurocranium excluding face. Obviously, we have actually measured the volume of brain case and scalp. We fully realize the limitations of such a technique however sequential changes in such measurements would mean actual changes of volume of neurocranium and scalp. In our method the inferior surface of the neurocranium-scalp was defined by two intersecting planes, one by the eyebrows and point of attachment of pinnas to scalp and the other, the inferior margin of the occiput identified by palpation and upper margin of the ear as described above. No attempts were made to further define the inferior margin of the brain using other techniques.

Postnatal neurocranial scalp shrinkage as measured by this method represents a relatively significant volume loss in each group. Such a volume loss may result from diminishing volume of the intracranial cavity, volume of the scalp or both. The suture score increase paralleling the head size decrease as described by Williams et al. (8) suggests that clearing of edema of the scalp is unlikely to have contributed to this shrinkage and that a diminished intracranial volume is more likely explanation. Internal biparietal diameters measured by ultrasound techniques showed significant decrease (7) during the first few days of life further supporting this hypothesis. Loss of intracranial water or solids could result in the shrinkage of intracranial volume. The depletion of brain solids which comprises only $9-16 \%$ of intracranial weight seems unlikely (3). The likely explanation is the loss of cerebrospinal fluid or interstitial brain water. Preterm infants have been shown to have more interstitial brain water than term infants (3). This could explain the relative changes in the cranial volume between these two groups. The differences in feeding patterns between term and preterm infants might also be responsible for the differences. Preterm infants are introduced to oral feedings more slowly than term infants and therefore reach equivalent fluid intakes much later. It is possible that tissue accretion rates may also differ between these two groups. In cases of sick preterm infants these differences may even be higher.

In conclusion, significant decreases in skull volume and OFC were seen in preterm infants for the first $160 \mathrm{~h}$ of age. In term infants only cranial volume was decreased. The OFC measurements may be misleading in the first week of life in term and preterm infants if it is used to assess brain growth.

\section{REFERENCES AND NOTES}

1. Bray, P. F., Shields, W. D., and Wolcott, G. J.: Occipitofrontal head circumference-An accurate measure of intracranial volume. J. Pediatr., 75: 303 (1969).

2. Buda, F. B., James, B., and Rabe, E. F.: Skull volumes in infants. Am. J. Dis. Child., 129: 1121 (1975).

3. Friis-Hansen, B.: Body composition during growth. Pediatrics, 47 (Suppl: 264 (1971).

4. Klaus, M. H. and Fanaroff, A. A.: Care of high risk neonates, 2nd Edition. pp. 113 (W. B. Saunders Co., Toronto, 1979)

5. Mackinnon, I. L., Kennedy, J. A., and Davies, T. V.: The estimation of skull capacity from roentgenologic measurements. Am. J., Roentgenol. Radium. Ther. Nucl. Med., 76: 303 (1956).

6. Maclaurin, J. C.: Changes in body water distribution during the first two weeks of life. Arch. Dis. Child., 41: 286 (1966)

7. Valkeakari, T: Changes of neonatal head diameter during the first week of life. Ann. Chir. Gynecol. Fenn., 64: 176 (1975).

8. Williams, J., Hirsch, N. J., Corbet, A. J. S., and Rudolph, A. J.: Postnatal head shrinkage in small infants. Pediatrics, 59:619 (1977).

9. Many thanks to the nurses at the Neonatal Nursery for their excellent care, to Professor Brian Habbick for reviewing the manuscript and to Louise Valade for secretarial assistance. Part of this experiment was supported by a grant from the Saskatchewan Health Research Board.

10. Requests for reprints should be addressed to: Dr. K. Sankaran, Director, Newborn Intensive Care Unit, University Hospital, Saskatoon, Saskatchewan, Canada.

11. Received for publication October $4,1982$.

12. Accepted for publication April 6,1983 . 